\section{Beispiel einer Auswertung ${ }^{10}$}

Es stehen vorerst nur Messungen der Kuns-Koeffizienten $K$ zur Verfügung, so daß die Auswertung nach Verfahren a) des vorigen Kapitels vorgenommen werden muß. Abb. 2 zeigt für $p$-Dimethylamino$p^{\prime}$-nitro-stilben in Benzol bei $20{ }^{\circ} \mathrm{C}$ den Kunsschen dichroitischen Koeffizienten $K$ als Funktion von $\mathrm{d} \log \varepsilon_{0} / \mathrm{d} \tilde{\boldsymbol{v}}$ für vier Wellenlängen, die alle innerhalb der ersten Absorptionsbande liegen. Die nach (25) $\mathrm{zu}$ erwartende lineare Abhängigkeit ist innerhalb der Meßfehler erfüllt. Mit $\mu_{\mathrm{g}}=7,14 \mathrm{D}$ erhält man aus Achsenabschnitt und Anstieg dieser Geraden $\gamma=16^{\circ}$, damit nach Gln. (38) und (39) $\beta=11^{\circ}$, $\mu_{\mathrm{a}}=23 \mathrm{D}$. Letzterer Wert stimmt innerhalb der Fehlergrenze mit dem aus der elektrischen Fluoreszenzpolarisation erhaltenen $\mu_{\mathrm{a}}(21,9 \mathrm{D})$ überein.



Abb. 2. KUнN-Koeffizienten für $p$-Dimethylamino- $p^{\prime}$-nitrostilben in Benzol bei $20^{\circ} \mathrm{C}$ als Funktion des Anstiegs der logarithmischen Absorptionskurve [Anwendung der Gl. (25)].

Wir danken Herrn Professor G. BRIegleb herzlich für die Förderung dieser Arbeit.

\title{
Verfahren zur Bestimmung der Konzentration von Radikalen durch Elektronenspinresonanz
}

\author{
Von G. v. Foerster \\ Aus dem Physikalischen Institut der Universität Gießen \\ (Z. Naturforschg. 15 a, 1079-1081 [1960]; eingegangen am 1. August 1960)
}

\begin{abstract}
Die Anzeigeempfindlichkeit einer Elektronenspinresonanzapparatur läßt sich dadurch ermitteln, daß zusätzlich zu der zu messenden Probe eine Eichprobe bekannter Spinzahl an eine geeignete Stelle im Resonator gebracht wird.
\end{abstract}

Durch Strahleneinwirkung auf Materie können freie Radikale produziert werden, zu deren quantitativer Bestimmung man sich mit Vorteil der Elektronenresonanzspektroskopie im Mikrowellengebiet bedient. Eine Reihe der heute bekannten Methoden ist hinsichtlich der Meßgenauigkeit und der Einfachheit noch keineswegs befriedigend. Im folgenden werden zwei Methoden beschrieben, die eine Bestimmung der Zahl der in einer Meßprobe enthaltenen freien Radikale relativ zu einem bekannten Normal zulassen. Störende Eigenschaften der Probe und ihre Rückwirkungen auf die Apparatur können eliminiert werden.

Ein Maß für die Radikalkonzentration gibt die Fläche unter dem Resonanzsignal. Jedoch gehen noch eine Reihe weiterer Parameter ein: Güte und Resonanzfrequenz des Resonators, Belastung der Mikrowellendiode und Abgleich der Mikrowellenapparatur. Eine Bestimmung der Empfindlichkeit der Apparatur

\footnotetext{
1 A. Ehrenberg u. L. Ehrenberg, Ark. Fysik 14, 133 [1958].
}

durch Austausch der zu messenden Substanz gegen eine Eichsubstanz führt daher nicht zum Ziel. Vielmehr muß die Empfindlichkeit bei Belastung durch die zu messende Probe bestimmt werden.

Bei einer bisher bekannten Methode ${ }^{1}$ wird die Empfindlichkeit der unbelasteten Apparatur festgestellt und durch Messung von Proben verschiedener Masse mit anschließender Extrapolation der erhaltenen Werte auf sehr kleine Masse die unbekannte Spinzahl ermittelt. Leider wird aber bei kleinen Spinzahlen die Meßgenauigkeit kleiner. In einem anderen Verfahren ${ }^{2}$ wird die Empfindlichkeit der Apparatur durch gleichzeitiges Registrieren des Spektrums der Meßprobe und der Eichprobe bestimmt. Um die Resonanzabsorption der beiden Proben zu trennen, wird das Magnetfeld am Ort der Vergleichsprobe durch Eisenscheibchen etwas verändert.

Zur Untersuchung umfangreichen biologischen Materials ergab sich für uns die Notwendigkeit einer

2 W. Köhnlein u. A. Müller, Z. Naturforschg. 15 b, 138 [1960]. 
genauen und schnellen Messung der Radikalkonzentration. An der Elektronenspinresonanzapparatur, welche bei $3,2 \mathrm{~cm}$ Wellenlänge arbeitet ${ }^{3}$, wurde dazu der Resonator konstruktiv verändert. Es bewährten sich die zwei folgenden Verfahren:

a) Es wird mit der zu messenden Substanz eine geeignete Vergleichssubstanz mitregistriert, die wegen einer Kernhyperfeinstruktur ein Linienspektrum von sechs äquidistanten Linien besitzt. Daher erübrigt sich die Verwendung von Eisenscheibchen am Resonator, um Meß- und Vergleichsspektrum zu trennen. Abb. 1 zeigt die Resonanzabsorption von Bohnenschalen (Vicia faba var. minor, Wassergehalt $4,5 \%$ ), die vier Tage dem Licht von Leuchtstoffröhren (ca. 4500 Lux) ausgesetzt waren. Die seitlichen Resonanzlinien stammen von der Vergleichsprobe.

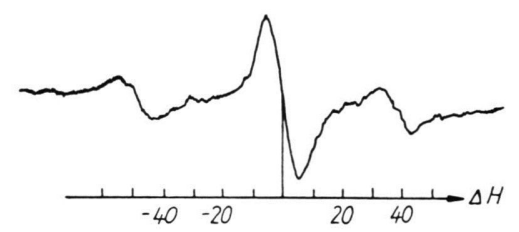

Abb. 1. Registrierkurven der paramagnetischen Resonanz von Bohnenschalen $(4,5 \%$ Feuchtigkeitsgehalt $)$ nach viertägiger Beleuchtung mit ca. 4500 Lux. Zu beiden Seiten HFS-Komponenten der Vergleichsprobe.

Die Höhe einer mitregistrierten Linie der Vergleichssubstanz ist ein Maß für die Anzeigeempfindlichkeit der Apparatur. Die Spinzahl der Vergleichssubstanz braucht nicht bekannt zu sein. Lediglich einmal wird die Vergleichssubstanz (in unserem Falle Magnesiumarsenat 054 der Osram-Studiengesellschaft) gemeinsam mit einer Eichprobe von DPPH registriert und so die Höhe der Vergleichssubstanzabsorption an die Resonanzabsorption des DPPH angeschlossen. Die Vergleichsprobe wird an eine Stelle geringer magnetischer Wechselfeldstärke in den Resonator eingeführt (Abb. 2). Sie kann daher unbedenklich, ohne Rückwirkung auf die Frequenz der Apparatur, gegen eine kleine Probe mit DPPH ausgetauscht werden. Dies wurde von uns dazu ausgenutzt, die Frequenz eines Protonenresonanzgenerators so einzustellen, daß sich die Kernresonanzlinie auf einem Doppelstrahloszillographen genau unter der DPPH-Absorptionslinie befand. Auch nach Entfernung des DPPH aus dem Resonator konnte dann der g-Wert des DPPH in das Meßspektrum eingefügt werden.

3 W. Stieler, Z. angew. Phys. 10, 89 [1958].

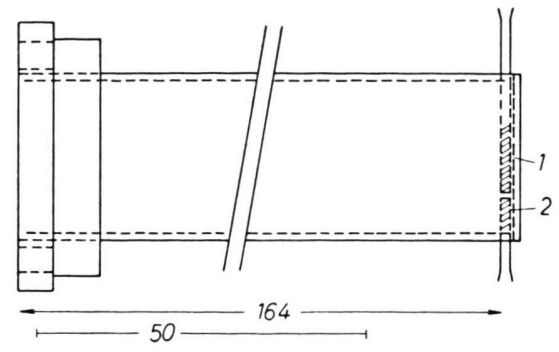

Abb. 2. Hohlraumresonator $\mathrm{H}_{107}$. 1. Ort der Proben $M$ bzw. $D_{1}$. 2. Ort der Probe $V$ bzw. $D_{2}$.

Das Verfahren ist auf solche Fälle beschränkt, bei denen das Meßspektrum genügend schmal ist und sich nicht zu sehr mit dem des Vergleichsspektrums überlappt. Diese Einschränkung entfällt im nächsten Verfahren bei unveränderter apparativer Anordnung.

b) Eine Eichprobe $D_{1}$ (eine abgewogene Menge DPPH) oder die zu messende Substanz $M$ werden wahlweise in den Resonator an die gleiche Stelle größter magnetischer Hochfrequenzfeldstärke eingeführt (s. Abb. 2). An eine andere, unempfindliche Stelle des Resonators mit kleiner magnetischer Hochfrequenzfeldstärke kann eine Vergleichsprobe mit DPPH $\left(D_{2}\right)$ gebracht werden. Es werden dann auf dem Oszillographenschirm die maximalen Signalamplituden $U$ gemessen.

Um die Empfindlichkeit der Apparatur zu bestimmen, wird zunächst

1)

$$
U\left(D_{1}\right)
$$

gemessen. Nach Hinzufügen der Probe $D_{2}$ ergibt sich:

$$
U\left(D_{1}+D_{2}\right) .
$$

Der Signalzuwachs durch die Probe $D_{2}$ beträgt also :

$$
\Delta U_{\mathrm{E}}=U\left(D_{1}+D_{2}\right)-U\left(D_{1}\right) .
$$

Diese Messung wird nur ein einziges Mal durchgeführt. Die oszillographische Registrierung der Meßprobe ergibt

3)

$$
U(M)
$$

und nach Hinzufügung der Probe $D_{2}$ :

$$
U\left(M+D_{2}\right) \text {. }
$$

Der Signalzuwachs durch die Probe $D_{2}$ kann in diesem Falle ein anderer sein:

$$
\Delta U_{\mathrm{MI}}=U\left(M+D_{2}\right)-U(M) .
$$

Die Messungen 3) und 4) werden jeweils vor jeder Registrierung eines Meßspektrums vorgenommen. 
Zur Bestimmung der Empfindlichkeit des Schreibers relativ zu der des Oszillographen wird einmal die Registrierung der Probe $D_{1}$ mit dem Schreiber vorgenommen. Dazu wird zur Vermeidung von Übersteuerungen des phasenempfindlichen Gleichrichters vorübergehend die Verstärkung des Vorverstärkers um einen Faktor $G$ reduziert.

Da bei der Messung 2) und 4) sich die gleiche Probe $D_{2}$ an der gleichen Stelle im Resonator befindet, gibt die ihr zugeordnete Signalhöhe den Einfluß der Meßprobe auf die Empfindlichkeit der Apparatur wieder. Der Quotient

$$
\Delta U_{\mathrm{M}} / \Delta U_{\mathrm{E}}
$$

ist das Verhältnis der Empfindlichkeiten der Apparatur belastet mit der Meßprobe bzw. mit der Eichprobe. Durch Berücksichtigung dieses Faktors werden sämtliche Veränderungen der Apparatur erfaßt, die bis zum Ausgang des Vorverstärkers auftreten können: Güteänderungen des Resonators, Änderungen des Brückenabgleichs ${ }^{4}$, der Klystronleistung, der Belastung der Diode, der Verstärkung des Vorverstärkers.

Ein Maß für die Spinzahl einer Meßprobe ist die Fläche unter ihrer Resonanzabsorptionskurve. Diese kann durch Integration aus der experimentell durch den Schreiber registrierten 1. Ableitung der Resonanzabsorptionskurve erhalten werden.

Werden Meßprobe und Eichprobe mit verschiedenen Wechselfeldamplituden $\tilde{H}_{\mathrm{M}}$ und $\tilde{H}_{\mathrm{E}}$ abgetastet, so gilt unter der Voraussetzung differentieller $\mathrm{Ab}$ tastung für die Spinzahl der Meßprobe:

$$
\text { I: } \quad N_{\text {Probe }}=\frac{\int_{-\infty}^{+\infty}\left(\int_{0}^{H} \frac{S_{\mathrm{M}}(\bar{H})}{\tilde{H}_{\mathrm{M}}} \mathrm{d} \bar{H}\right) \mathrm{d} H}{G \cdot \int_{-\infty}^{+\infty}\left(\int_{0}^{H} \frac{S_{\mathrm{E}}(\bar{H})}{\tilde{H}_{\mathrm{E}}} \mathrm{d} \bar{H}\right) \mathrm{d} H} \frac{\Delta U_{\mathrm{E}}}{\Delta U_{\mathrm{M}}} N_{\text {DPPH }} \text {, }
$$

wobei $S_{\mathrm{M}}$ bzw. $S_{\mathrm{E}}$ den Verlauf der Schreiberregistrierungen der Meßprobe $M$ bzw. der Eichprobe $D_{1}$ bedeuten.

Man kann auf die Integration verzichten, wenn die Resonanzkurven der Meßprobe und der Eichprobe die gleiche Kurvenform besitzen. Dann läßt sich die Fläche $F$ unter der Resonanzabsorptionskurve darstellen durch

$$
F=\gamma \frac{S_{\max }}{\tilde{H}}(\Delta H)^{2},
$$

${ }^{4}$ G. v. Foerster, Diplomarbeit, Gießen 1959. wobei $\gamma$ ein für beide Kurven gleicher Formfaktor ist. Die auf dem Schreiber angezeigte Maximalspannung $S_{\max }$ ist proportional der maximalen Steigung der Resonanzkurve. Gl. I geht dann über in

$$
\text { II: } \quad N_{\text {Probe }}=\frac{\frac{S_{\mathrm{M} \max }}{\tilde{H}_{\mathrm{M}}}\left(\Delta H_{\mathrm{M})^{2}}\right.}{G \frac{S_{\mathrm{E} \max }}{\tilde{H}_{\mathrm{E}}}\left(\Delta H_{\mathrm{E}}\right)^{2}} \frac{\Delta U_{\mathrm{E}}}{\Delta U_{\mathrm{M}}} N_{\mathrm{DPPH}} \text {. }
$$

Wählt man experimentell die Wechselfeldamplituden proportional den jeweiligen Linienbreiten,

$$
\tilde{H}_{\mathrm{M}}=\alpha \Delta H_{\mathrm{M}}, \quad \tilde{H}_{\mathrm{E}}=\alpha \Delta H_{\mathrm{E}},
$$

so darf man die Voraussetzungen differentieller Abtastung fallen lassen und erhält

$$
\text { III : } \quad N_{\text {Probe }}=\frac{\Delta H_{\mathrm{M}} S_{\mathrm{M} \max }}{G \Delta H_{\mathrm{E}} S_{\mathrm{E} \max }} \frac{\Delta U_{\mathrm{E}}}{\Delta U_{\mathrm{M}}} N_{\mathrm{DPPH}} \text {. }
$$

Auf die Formeln II und III wird man im allgemeinen nur zurückgreifen, wenn die Integration der Meßkurven wegen zu geringer Intensität des Spektrums problematisch wird.

Die vorstehenden Formeln ergeben die Spinzahl der Meßprobe unter der Voraussetzung, daß die Geometrie von Meßprobe und -küvette und Eichprobe und -küvette gleich ist. Wenn dies nicht der Fall ist, muß der Verlauf der Empfindlichkeit im Resonator mit einer Sonde ausgemessen oder aus der Feldverteilung im Resonator ${ }^{5}$ berechnet und durch einen Korrekturfaktor berücksichtigt werden. Die bei der Umrechnung auf gleiche Probengeometrie auftretenden Fehler sind kleiner als diejenigen, die bei der Herstellung einer Eichprobe DPPH mit der Meßprobe gleicher Geometrie auftreten würden. Nach Anbringung sämtlicher Korrekturen erhält man Spinzahlen, die sich auf die in der Eichsubstanz angegebene Spinzahl beziehen. Zur Angabe der Spinzahl der Meßprobe gehört also noch die Angabe, mit welcher Eichsubstanz sie ermittelt wurde, weil man heute noch nicht sicher ist, ob z. B. DPPH vollständig als Radikal vorliegt. Mit der beschriebenen Methode lassen sich Spinzahlen auf einige Prozent genau relativ zur Eichsubstanz angeben.

Herrn Prof. Dr. W. HANLe und Herrn Prof. Dr. A. Schmillen danke ich für wertvolle Diskussionen. Herr Dipl.-Biol. Dr. W. Klingmüller stellte freundlicherweise die Meßsubstanz für Abb. 1 zur Verfügung. Der D e u t $\mathrm{schen} \mathrm{Forschungsgemeinschaft} \mathrm{danke} \mathrm{ich}$ für die Bereitstellung von Sachmitteln.

5 Meinke-Gundlach, Taschenbuch der Hochfrequenztechnik, Springer-Verlag, Berlin 1956, S. 225. 